\title{
In Utero MR Imaging of Fetal Holoprosencephaly: A Structured Approach to Diagnosis and Classification
}

(D)P.D. Griffiths and (DD. Jarvis

\begin{abstract}
BACKGROUND AND PURPOSE: Holoprosencephaly is a rare developmental brain abnormality with a range of severity. We describe our experience in diagnosing holoprosencephaly in the fetus with in utero MR imaging. We hypothesized that including in utero MR imaging in the diagnostic pathway will improve the detection of holoprosencephaly compared with ultrasonography and allow better assessment of the severity.
\end{abstract}

MATERIALS AND METHODS: We report on holoprosencephaly identified from ultrasonography and/or a diagnosis of holoprosencephaly made with in utero MR imaging. We compare the diagnoses made with sonography and in utero MR imaging in each case and compare the 2 methods of assessing the severity of holoprosencephaly.

RESULTS: Thirty-five fetuses are reported, including 9 in which the diagnosis of holoprosencephaly was made on ultrasonography but not confirmed on in utero MR imaging. Of the 26 cases of holoprosencephaly diagnosed on in utero MR imaging, 12 were not recognized on ultrasonography.

CONCLUSIONS: Our results show that in utero MR imaging has a major role in diagnosing or refuting a diagnosis of fetal holoprosencephaly made on ultrasonography. In utero MR imaging also assists in grading the severity of fetal holoprosencephaly.

ABBREVIATIONS: HPE = holoprosencephaly; iUMR = in utero MR imaging; MIHF = middle interhemispheric fusion variety of holoprosencephaly; US = ultrasonography

n utero MR imaging (iuMR) is now an accepted method of diagnosing fetal brain abnormalities and is an important adjunct to the diagnostic pathway alongside antenatal ultrasonography (US). Ultrafast iuMR methods (broadly defined as acquiring 1 image per second) are required to get good-quality imaging in utero because of movement from maternal and fetal sources. Heavily weighted T2 techniques are the mainstay of imaging the fetal brain and are usually acquired with singleshot fast spin-echo sequences. The nonmyelinated or poorly myelinated fetal brain is best imaged with T2-weighted sequences because of the improved tissue contrast between the different components of the fetal brain, which is particularly marked in the second trimester. ${ }^{1}$ However, different methods

Received July 22, 2015; accepted after revision August 17.

From the Academic Unit of Radiology, University of Sheffield, Sheffield, UK.

Please address correspondence to Paul Griffiths, FRCR, Academic Unit of Radiology, Floor C, Royal Hallamshire Hospital, Glossop Rd, Sheffield S10 2JF, UK; e-mail: p.griffiths@sheffield.ac.uk

三 Indicates article with supplemental on-line table.

http://dx.doi.org/10.3174/ajnr.A4572 of acquiring T2-weighted images, such as steady-state sequences, offer other advantages. ${ }^{2,3}$

In this study, we describe our experience in applying these techniques in the diagnosis and classification of the severity of fetal holoprosencephaly (HPE). HPE is a relatively rare disorder with an estimated prevalence of 1 in 16,000 live births, though it is found in 1 of 250 spontaneous abortions. ${ }^{4} \mathrm{HPE}$ arises as a result of abnormal ventral induction, which causes impaired growth and failure of midline cleavage of the future cerebral hemispheres. The phenotype is exceptionally heterogeneous, and the clinical implications for a fetus/child are closely related to the severity of the malformation. The most severe forms of HPE are not consistent with long-term, extrauterine life, while the minor forms can produce relatively few symptoms.

We hypothesize that including iuMR in the diagnostic pathway will improve the detection of HPE compared with US and allow better assessment of the severity of HPE in an affected fetus.

\section{MATERIALS AND METHODS Study Population}

This is a retrospective study of pregnant women referred to our Institution from fetomaternal units in England and Scotland, 


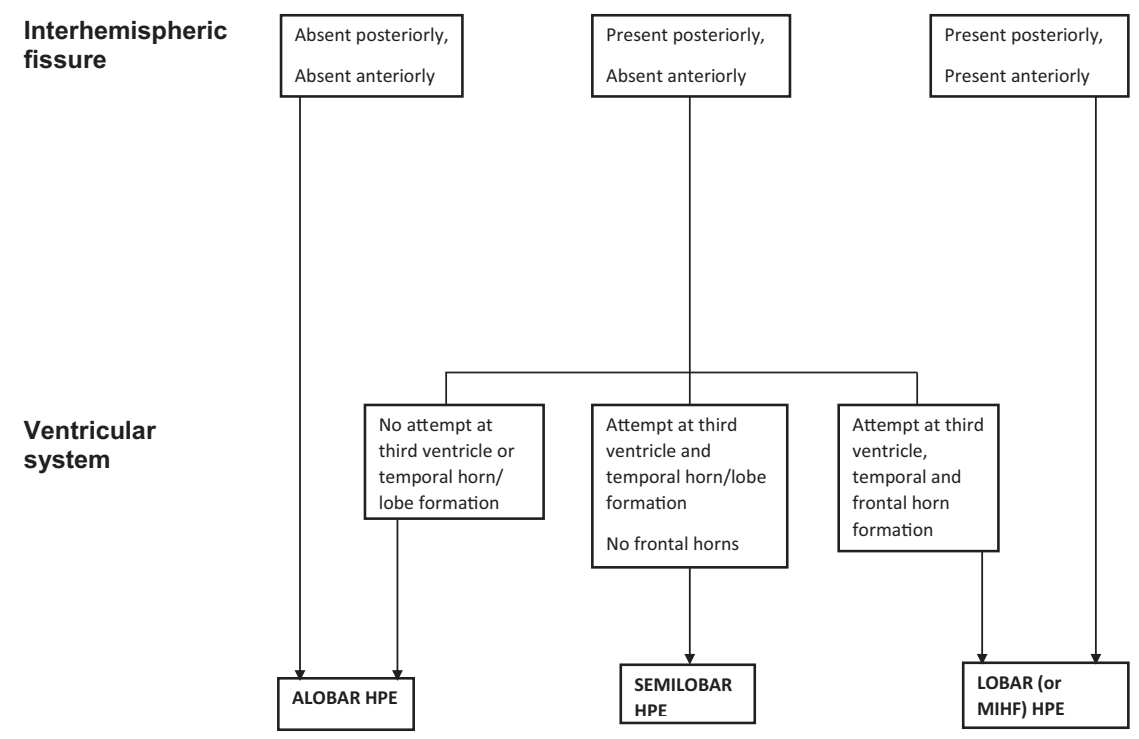

FIG 1. A flowchart showing the classification of alobar, semilobar, and lobar holoprosencephaly in this article.

whose fetuses were either suspected of having HPE on US and/or were shown to have HPE following iuMR. Appropriate cases were located on the MR imaging data base of the institution during a 15-year period (2000-2014). All cases had US performed by a fetal-maternal expert from 1 of 11 tertiary centers before referral to our Institution for their iuMR study, and the full sonography report was available to the radiologist performing the iuMR study. Women were screened for contraindications to MR imaging, and iuMR examinations were not performed before 18 weeks' gestational age. Of the 35 cases found on the data base, 23 women were scanned as recruits into wider research studies of fetal iuMR, and they all provided informed written consent under the guidance and approval of Research Ethics Committees. Seven out of 23 of the research cases were referred from the Magnetic Resonance Imaging to Enhance the Diagnosis of Fetal Developmental Brain Abnormalities in Utero study. ${ }^{5}$ These women were not paid for their involvement in the study, but travel expenses were offered. Relevant review was sought, and approval was obtained from the Institutional Clinical Effectiveness Unit and Research Department to allow the 12 cases with imaging performed for clinical purposes to be reported in this article as well.

\section{MR Imaging}

In utero MR imaging was performed on whole-body $1.5 \mathrm{~T}$ scanners (before 2008, Infinion; Philips Healthcare, Best, Netherlands; from 2008 onward, HDx; GE Healthcare, Milwaukee, Wisconsin). Maternal sedation was not used for any of the scans. The iuMR protocol changed during the course of the study as new methods became available; however, all subjects had ultrafast T2-weighted images of the fetal brain in the 3 orthogonal planes by using single-shot fast spinecho sequences (4- to 5-mm thickness). Ultrafast T1-weighted and diffusion-weighted imaging in the axial plane were acquired routinely from 2007; and after 2011, 3D datasets were added by using a steady-state sequence (3D FIESTA). The imaging parameters for the $3 \mathrm{D}$ sequence are described in detail elsewhere ${ }^{2}$ but are summarized here: TR minimum, 4-5 ms; TE minimum, 2-3 ms; refocusing flip angle, $60^{\circ}$; NEX, 0.75; FOV, $340 \times 270 \mathrm{~mm}$; matrix size, $320 \times 256 \mathrm{~mm}$. The partition thickness varied between 1.8 and $2.2 \mathrm{~mm}$ with 28-32 scan locations per slab to allow full coverage of the fetal brain with maximum resolution; this was achieved in 18- to 23second acquisitions.

The 3D datasets were transferred to a desktop PC and loaded into 3D Slicer software (http://www.slicer.org) for segmentation. This is an open source image processing and analysis software package. Each fetal brain was segmented manually by outlining the outer surface and ventricular margins on a section-by-section basis by a single trained operator (D.J.) with input from a neuroradiologist experienced in fetal brain imaging (P.D.G.) and with reference to a fetal brain atlas. ${ }^{1}$ Segmentation took approximately 60 minutes for a second trimester fetal brain and 90 minutes for more mature fetuses; the longer time was due to the increased complexity of sulcation/gyration. Reconstruction of that data allowed the creation of surface representations of the whole fetal brain and ventricular system.

\section{Analysis}

All of the iuMR examinations were reported at the time of the study but were reviewed for the purpose of this study by a pediatric neuroradiologist experienced in fetal brain MR imaging (P.D.G.). In cases in which the diagnosis of HPE was not supported by the iuMR findings, the alternative diagnoses were recorded. Further detailed systematic anatomic analyses were made in the cases in which HPE was present on iuMR.

\section{DeMyer Classification}

The first analysis was to ascribe a diagnosis of alobar, semilobar, and lobar HPE, based on the original descriptions of DeMyer and Zeman $^{6}$ and DeMyer, ${ }^{7}$ and in the neuroradiologic literature of Simon et $\mathrm{al}^{8}$ and Barkovich et al, ${ }^{9}$ with some minor modifications (Fig 1). Three cases of fetal HPE did not fit into that standard description of ventral HPE but were classified as middle interhemispheric fusion varieties of HPE (MIHF). This variant was not described in the original works of DeMyer, ${ }^{6,7}$ and our classification is based on the criteria suggested by Simon et al. ${ }^{10}$

\section{Severity Score}

A more detailed secondary anatomic analysis was made by assessing the parts of the brain frequently affected by HPE, broadly following the approach of Simon et $\mathrm{al}^{8}$ and Barkovich et $\mathrm{al}^{9}$ in children. It was necessary to make some modifications to those assessments to customize them for fetal brains. These involved some simplification of the methods for reasons detailed in the "Discussion." "Present" or "absent" assessments were made of the posterior interhemispheric fissure, posterior falx, anterior inter- 
Antenatal US and iuMR information in 9 cases in which fetal holoprosencephaly had been diagnosed on US but was not confirmed on iuMR

\begin{tabular}{|c|c|c|c|}
\hline Case & $\begin{array}{l}\text { Gestational Age at } \\
\text { iuMR (wks) }\end{array}$ & Antenatal US Findings & iuMR Findings \\
\hline A & 24 & Lobar HPE & Septo-optic dysplasia, schizencephaly \\
\hline B & 22 & HPE (severity not specified) & Agenesis of corpus callosum \\
\hline C & 24 & HPE (severity not specified) & $\begin{array}{l}\text { Ventriculomegaly, disrupted cavum septum pellucidum probably secondary } \\
\text { to hydrocephalus }\end{array}$ \\
\hline D & 27 & HPE (severity not specified) & Septo-optic dysplasia, schizencephaly \\
\hline E & 21 & HPE (severity not specified) & Agenesis of corpus callosum \\
\hline $\mathrm{F}$ & 27 & Lobar HPE & Isolated absence of cavum septum pellucidum \\
\hline G & 18 & HPE (severity not specified) & $\begin{array}{l}\text { Ventriculomegaly, disrupted cavum septum pellucidum probably secondary } \\
\text { to hydrocephalus }\end{array}$ \\
\hline $\mathrm{H}$ & 21 & Lobar HPE & Septo-optic dysplasia, schizencephaly \\
\hline 1 & 24 & HPE (severity not specified) & $\begin{array}{l}\text { Ventriculomegaly, disrupted cavum septum pellucidum probably secondary } \\
\text { to hydrocephalus }\end{array}$ \\
\hline
\end{tabular}

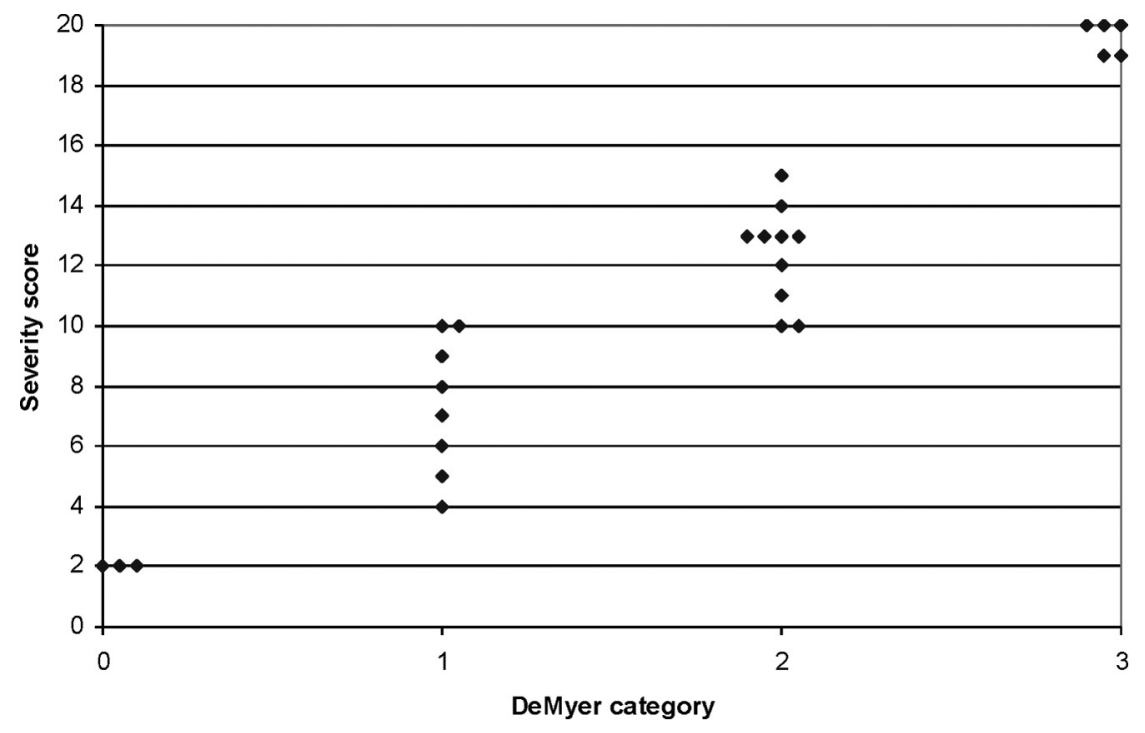

FIG 2. Scatterplot showing the correlation between the DeMyer classification and the severity score in 26 cases of fetal holoprosencephaly.

hemispheric fissure, anterior falx, third ventricle, temporal horns/ lobes, and frontal horns. The structures did not have to be complete; some attempt at formation was sufficient to be scored as zero (present), whereas if the structure was not present at all, it was scored as 1 . Similar binary scores were awarded for the hypothalamus (complete separation $=0$, noncleavage $=1$ ), the midbrain (completely separated from the diencephalon $=0$, some degree of nonseparation $=1$ ), and the orbital portions of the frontal lobe, dorsomedial portions of the frontal lobes, and paracentral lobes (complete separation $=0$, noncleavage $=1$ for each of those regions). Assessments of the deep gray structures of the cerebral hemispheres (caudate, lentiform, and thalamic nuclei) were performed independently on a 3-point scale (as opposed to a 4 -point scale by Simon et $\mathrm{al}^{8}$ ): complete separation $=0 ;<50 \%$ noncleavage or abnormally medially placed $=1$; and $\geq 50 \%$ noncleavage $=2$. A similar 3-point score was used to assess the corpus callosum: completely present $=0 ;>50 \%$ present but not fully formed $=1$; and $<50 \%$ present or absent $=2$. The scores were added together to provide a severity score with a total of 20 points for each fetus. Note that the cavum septum pellucidum was not used in this analysis because it was absent in all cases and was considered nondiscriminatory.

\section{RESULTS}

In $9 / 35$ cases $(26 \%)$, the diagnosis of HPE made on US was not supported by iuMR, and these cases are summarized in the Table. In all of these 9 cases, the cavum septum pellucidum was not demonstrated on US. On iuMR, the cavum septum pellucidum was shown to be either absent ( 4 cases, 1 with apparent isolated absence of the cavum septum pellucidum and 3 with possible septooptic dysplasia based on a boxlike deformity of the frontal horns); disrupted (3 cases associated with ventriculomegaly probably due to hydrocephalus); or abnormally situated ( 2 cases of agenesis of the corpus callosum).

\section{DeMyer Classification}

Twenty-six cases of HPE of any severity were depicted by iuMR, and a summary of the antenatal US and iuMR details of these cases is shown in the On-line Table. Most of these cases had relatively small head sizes. The median gestational age at the time of iuMR in that group was 21 weeks (interquartile range, 20-23 weeks; full range, 18-30 weeks). There were 5 cases of alobar HPE, 10 cases of semilobar HPE, and 8 cases of lobar HPE on iuMR based on the DeMyer and Zeman ${ }^{6}$ and DeMeyer ${ }^{7}$ classification and 3 cases of MIHF as described by Simon et al. ${ }^{10} \mathrm{~A}$ diagnosis of HPE was made by US in 23 fetuses (all 9 fetuses in the Table and 14 fetuses in the On-line Table); and in 9/23 cases (39\%), the diagnosis of HPE was not supported on iuMR (all those in the Table). A diagnosis of HPE was made on iuMR in 26 cases (all of those in the On-line Table); and in 12/26 cases (46\%), HPE was not recognized on US.

In the 14 cases in which HPE was diagnosed by both US and iuMR, 8/14 US reports did not specify the severity of HPE. In $3 / 14$ cases, the same severity was reported on both methods, and in $3 / 14$ cases, the severity diagnosed by US and iuMR was different. The agreement in diagnosing HPE between US and iuMR was related to the severity of HPE, specifically ultrasonography diagnosed more cases of the more severe forms of 

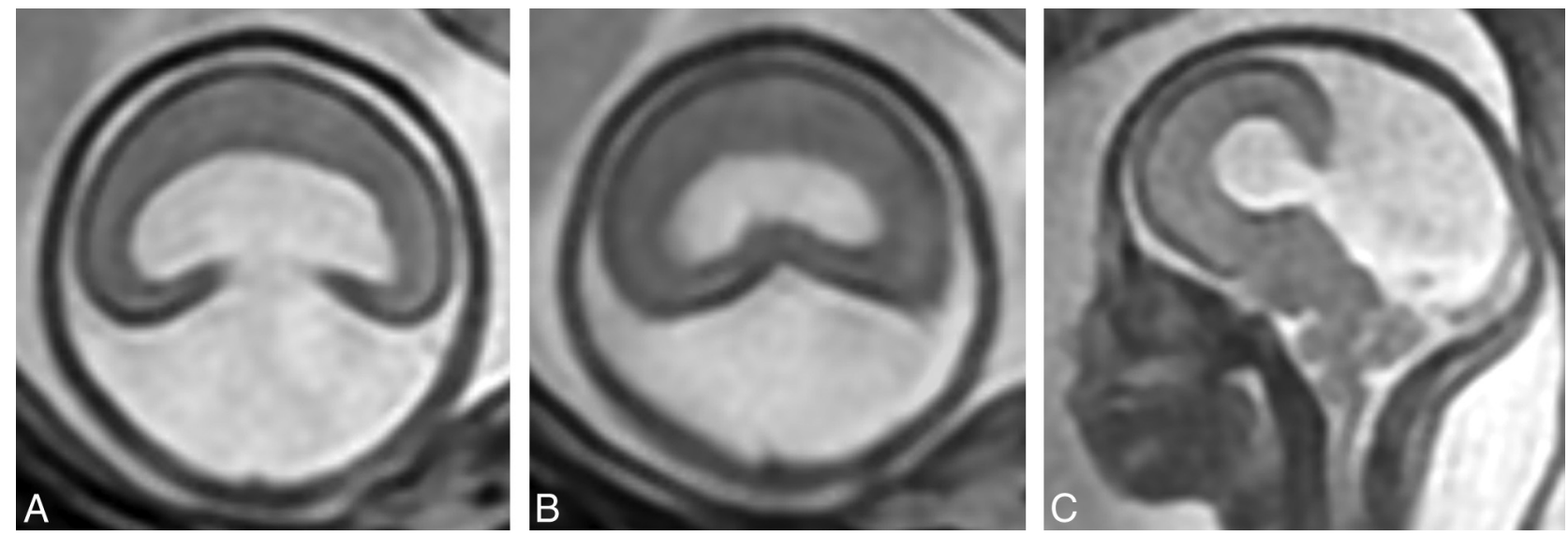

FIG 3. T2-weighted images (single-shot fast spin-echo) in a 24-week gestational age fetus with alobar holoprosencephaly according to the DeMyer classification and a severity score of 20/20 (case 2). Axial images of the supratentorial brain ( $A$ and $B$ ) show no attempt at formation of the interhemispheric fissure and a holoventricle and posterior cyst. Sagittal imaging (C) shows an abnormally kinked brain stem.
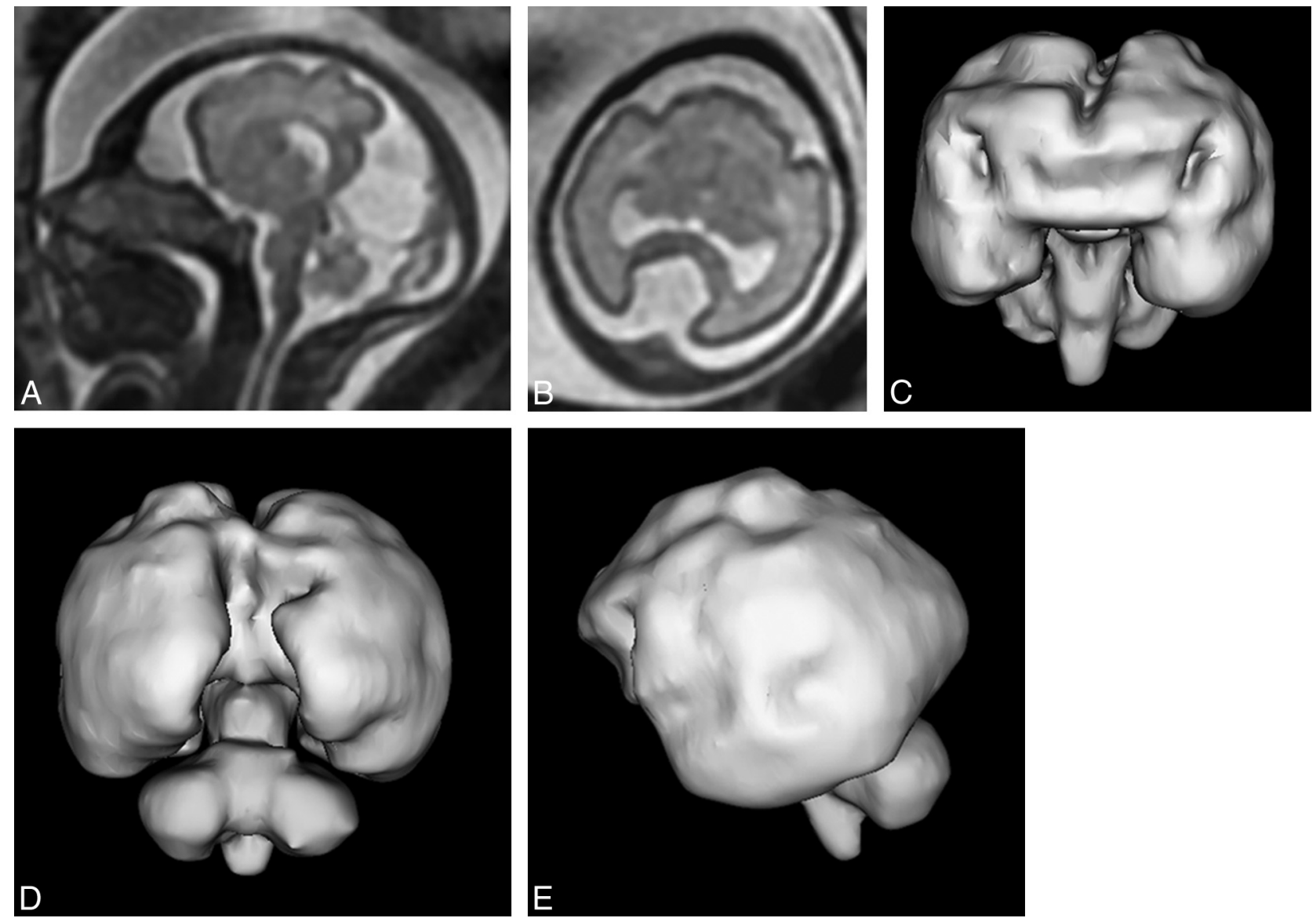

FIG 4. T2-weighted images (single-shot fast spin-echo, $A$ and $B$ ) and surface reconstructions of 3D steady-state (FIESTA) data of a 26week gestational age fetus with semilobar holoprosencephaly according to the DeMyer classification and a severity score of $13 / 20$ (case 19). Axial images of the supratentorial brain $(B)$ and posterior and anterior views of the brain $(C$ and $D)$ show some formation of the interhemispheric fissure posteriorly but not anteriorly. There is some attempt at formation of the third ventricle and temporal horns of the lateral ventricles. Lateral projections $(E)$ show abnormal sulcation of the cerebral hemispheres and marked frontal lobe hypoplasia.

HPE correctly. HPE was diagnosed on iuMR in 5/5 cases $(100 \%)$ of alobar HPE (US diagnoses: alobar $[n=3$ ], semilobar $[n=1]$, and not specified $[n=1])$. In the 10 cases of semilobar HPE diagnosed on iuMR, HPE was the given diagnosis on US in $4 / 10$ cases (40\%) (US diagnoses: not specified severity $[n=3$ ] and semilobar HPE $[n=1])$. In the 8 cases of lobar HPE diagnosed on iuMR, HPE was the given diagnosis on US in $4 / 8$ cases (50\%) (US diagnoses: not specified severity $[n=3]$ and semilobar HPE $[n=1])$. Only $1 / 3$ cases $(33 \%)$ of MIHF were recognized as HPE on US (called "lobar HPE”). 

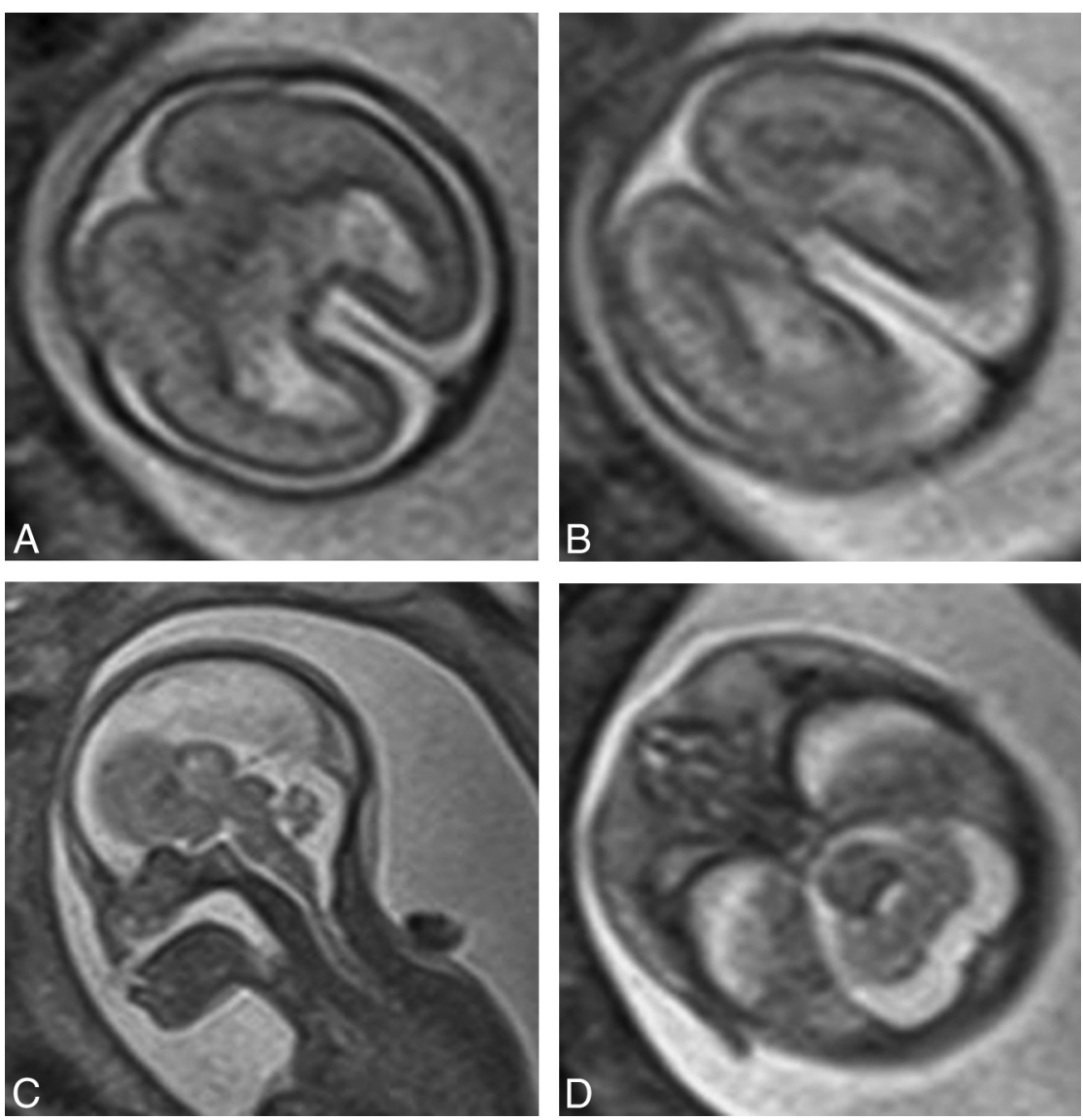

FIG 5. T2-weighted images (single-shot fast spin-echo) of a 21-week gestational age fetus with lobar holoprosencephaly according to the DeMyer classification and a severity score of $7 / 20$ (case 15). Axial images of the supratentorial brain ( $A$ and $B$ ) show formation of the interhemispheric fissure posteriorly and anteriorly and some attempt at formation of the frontal horns of the lateral ventricles. The frontal lobes are fused anteriorly and inferiorly. Despite the relatively low severity score, brain development is markedly deranged, as shown by the corpus callosum anatomy, abnormal opercularization, and brain biometry. There is also a significant facial malformation with absence of the nose $(C$ and $D)$ and a midline cleft lip/cleft palate.

\section{Severity Score}

The On-line Table shows the total severity scores based on the detailed assessment of the brain anatomy. There was a close correlation between the severity score and the DeMyer category of HPE as shown in Fig 2, and the importance of this is discussed in later sections. Representative images are shown in Figs 3-7.

\section{Other Brain Abnormalities}

Brain abnormalities other than HPE, not including face or intracranial vascular abnormalities, were shown on iuMR in 9/26 fetuses $(35 \%)$ with HPE. None of those were suspected on US. Other brain abnormalities were shown in $1 / 5$ fetuses with alobar HPE, 5/10 fetuses with semilobar HPE, 3/8 fetuses with lobar HPE, and 0/3 fetuses with MIHF. Structural abnormalities of the posterior fossa content were notable and included cerebellar hypoplasia, Dandy-Walker malformation, rhombencephalosynapsis and brain stem malformations.

\section{DISCUSSION}

HPE is a malformation that arises from abnormal ventral induction, a process that occurs 5-10 weeks after fertilization in hu- mans, during which there is failure of normal growth and cleavage of the cranial end of the neural tube. Hence, the primary effects are on the prosencephalon/future cerebral hemispheres. Abnormal ventral induction can produce a range of anatomic abnormalities of the brain, and the severity is closely linked to prognosis. Detectable chromosomal abnormalities are found in 24\%-45\% of cases of $\mathrm{HPE}^{4}$ and include numeric chromosomal abnormalities, most frequently trisomy 13 or 18 (only 1 case of a trisomy was demonstrated in our series, but not all fetuses had karyotyping). There is also an increased risk of HPE in fetuses with structural chromosomal abnormalities, particularly deletions of chromosomes 13, 18, and 7. HPE is thought to result from monogenic abnormalities in $18 \%-25 \%$ of cases, ${ }^{4}$ which can produce autosomal dominant disorders such as Pallister-Hall and RubinsteinTaybi syndromes, autosomal recessive disorders such as Smith-Lemli-Opitz and Meckel-Gruber syndromes ( 1 case in our series), or X-linked recessive disorders. In addition, there are a range of nongenetic associations that increase the risk of fetal HPE, including maternal diabetes and fetal exposure to retinoic acid, ethanol, and some anticonvulsants.

A woman whose pregnancy is complicated by possible HPE has a number of requirements from antenatal imaging, including the following: a confident diagnosis or exclusion of HPE, an accurate assessment of the anatomic severity of HPE, and a description of associated brain abnormalities. In the absence of consistent reference outcome data for our cases, all we can say with certainty is that there are major differences between the US and iuMR results. This comment needs to be balanced against the accumulating published data suggesting that iuMR is more accurate than US in diagnosing fetal brain pathology in general, as shown in a recent meta-analysis. ${ }^{11}$ Of the total 23 cases of US-diagnosed HPE described in this report, the chance of the diagnosis being incorrect was $39 \%$. Conversely, in the 26 cases in which iuMR diagnosed HPE of any severity, the chance of the diagnosis being missed on US was $46 \%$ if iuMR is assumed correct. US performed better in the more severe forms of HPE as might be expected. Our results also show that when HPE was correctly diagnosed on US as an overarching classification, the severity was often either incorrect (3/14) or not attempted (8/14).

The cases of fundamental disagreement about the diagnosis of HPE between US and iuMR warrant further discussion. The 9 cases in which HPE was diagnosed on US but was not confirmed on iuMR had 1 common feature: The cavum septum pellucidum was not visualized on US. Although the cavum septum pellucidum (septum pellucidum after birth) has little or no functional significance, it is a vital landmark for normal brain development 

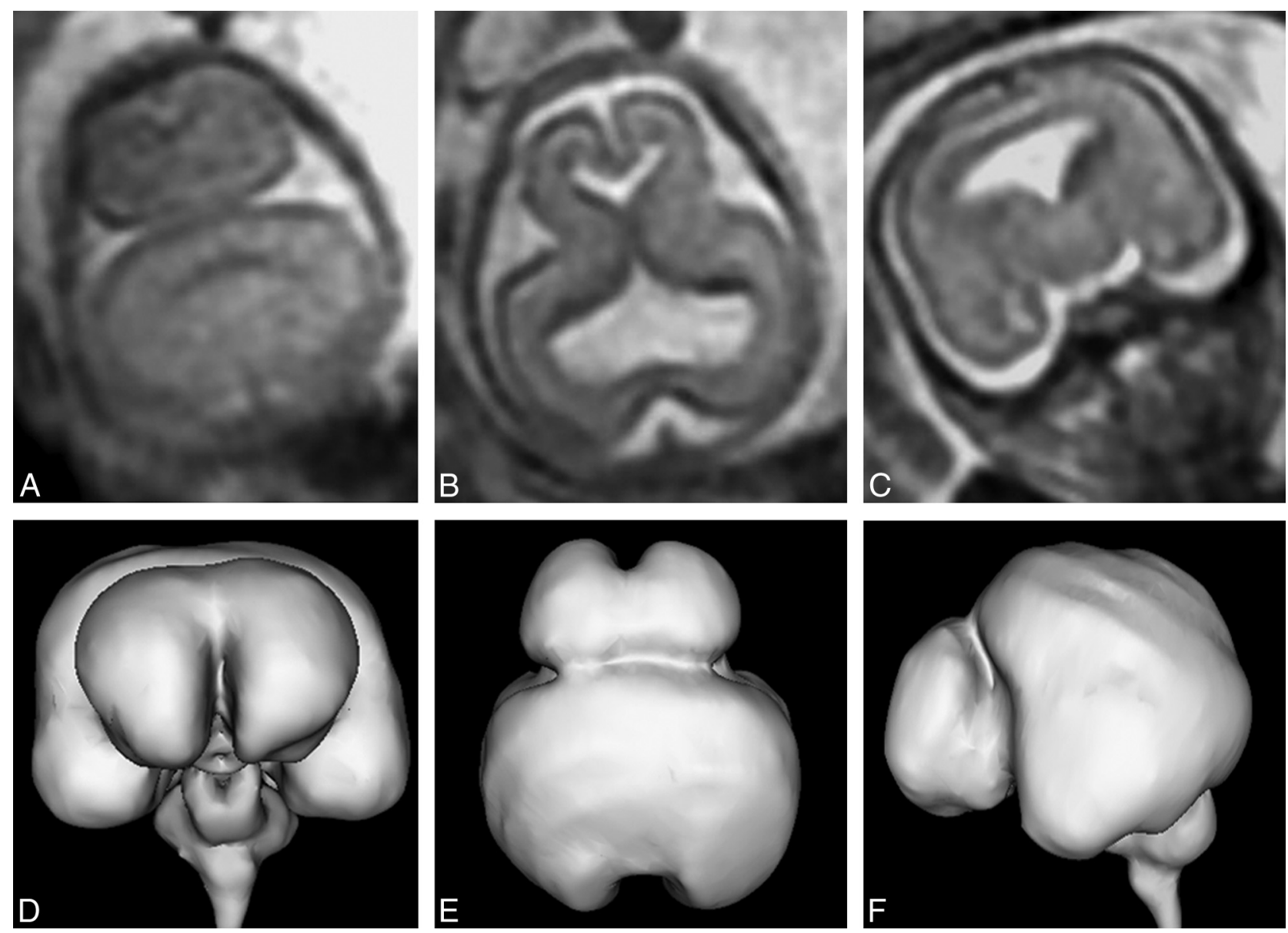

FIG 6. T2-weighted images (single-shot fast spin-echo, $A-C$ ) and surface reconstructions of 3D steady-state (FIESTA) data of the brain (D-F) in a 21-week gestational age fetus with lobar holoprosencephaly and a severity score of 9/20 (case 20). Axial images of the supratentorial brain ( $A$ and $B$ ) show interhemispheric fissure formation posteriorly and anteriorly. The third ventricle, temporal horns, and frontal horns of the lateral ventricles are separated. The coronal image $(C)$ shows fusion of the frontal lobes, thalamus, and hypothalamus. The case is complicated by marked frontal lobe hypoplasia and bilateral abnormal frontal sulci shown on the surface reconstructions $(D-F)$.

on antenatal and postnatal imaging. ${ }^{12,13}$ Nonvisualization of the cavum septum pellucidum is an imaging feature in all severities of HPE. However, it is also found in other brain abnormalities, and there are a number of different explanations for nonvisualization of the cavum septum pellucidum. It may be that the cavum septum pellucidum never formed (as in HPE, septo-optic dysplasia, and isolated absent cavum septum pellucidum), it may have formed but been destroyed (raised intraventricular pressure from hydrocephalus), or it may have formed but in an abnormal position (agenesis of the corpus callosum where the leaf of the septum pellucidum is often closely applied to the inferior surface of the ipsilateral Probst bundle). ${ }^{13,14}$ The alternative diagnoses on the basis of iuMR in the 9 false-positive cases all fit into these categories: The cavum septum pellucidum never formed 3 cases of septo-optic dysplasia and 1 case of isolated absent cavum septum pellucidum); or it formed but was secondarily disrupted (3 cases of ventriculomegaly probably due to hydrocephalus) or misplaced ( 2 cases of agenesis of the corpus callosum).

We also described 12 cases with false-negative findings in which the diagnosis of HPE on iuMR was not described on the referral from US. In 2 cases (cases 11 and 15), the diagnosis of HPE should have been strongly suspected from the US findings because of midline cleft lip and palate (1 with an absent cavum septum pellucidum), but in the other 10 cases, the diagnosis of
HPE on iuMR was unexpected. The US diagnoses varied in those 10 cases: 3 cases of agenesis or hypogenesis of the corpus callosum (cases 10, 19, and 24); 3 cases of isolated ventriculomegaly $(17,20$, and 25); 2 cases of posterior interhemispheric cyst (cases 18 and 26); 1 case of a posterior cephalocele (case 5); and 1 case of cerebellar hypoplasia (case 21). It is difficult to make any specific observations or teaching points from those cases.

There was a high rate of brain abnormalities other than HPE in our cohort-that is, in 9/23 (39\%) of the "classic" ventral HPE cases (ie, excluding the MIHF cases), most of which were cerebellar or brain stem malformations. No other brain abnormalities were found in the 3 cases of MIHF. If we assume that iuMR is more likely to provide the correct diagnosis, we suggest that there is a significant advantage in supplementing US with iuMR in the diagnostic pathway. This assumption is reasonable because though we are not aware of studies of diagnostic accuracy of iuMR, specifically in cases of HPE, the published literature suggests that it is true of fetal brain pathology in general as described above.

Our attempts to enhance the classification of fetal HPE were based on the previous work of Simon et $\mathrm{al}^{8,10}$ and Barkovich et $\mathrm{al},{ }^{9}$ who recognized the difficulty in apportioning a single DeMyer classification in pediatric cases of HPE. Their extensive experience and subsequent publications urge a more anatomically descrip- 

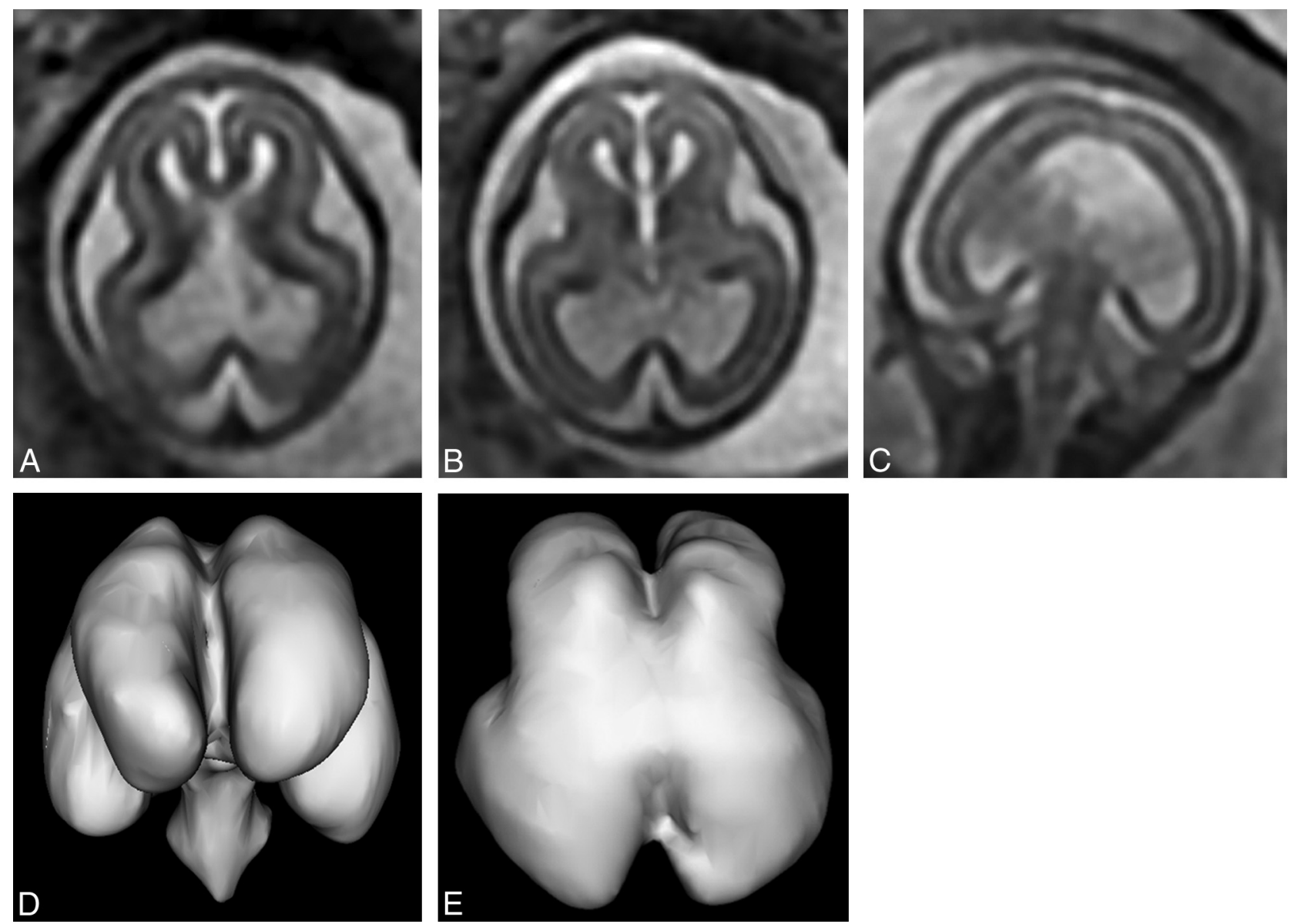

FIG 7. T2-weighted images (single-shot fast spin-echo, $A-C$ ) and surface reconstructions of 3D steady state (FIESTA) data of the brain ( $D$ and $E$ ) of a 19-week gestational age fetus with the interhemispheric fusion variety of holoprosencephaly and a severity score of $2 / 20$ (case 25 ). Axial images of the supratentorial brain $(A$ and $B)$ show complete separation of the interhemispheric fissure and central gray structures, but the ventricles are large and dysmorphic. Coronal imaging $(C)$ shows fusion of the paracentral lobules. These features are confirmed and better defined on the surface reconstructions.

tive approach, rather than relying on alobar, semilobar, and lobar classifications in pediatric practice. We attempted to reproduce their approach for fetuses in our study; there are several problems when one tries to apply their methods to iuMR, particularly in second trimester fetuses. Those authors most often used a 4-point scale to assess the sagittal separation of a particular anatomic structure: $0=$ complete separation; $1=$ minimal noncleavage or medial deviation; 2 = partial noncleavage; $3=$ total noncleavage. We found assessment in such detail exceptionally difficult because of the small size of the fetal structures and opted for either a binary assessment, present or absent, or a 3-point scale for the larger structures: completely present $=0 ;>50 \%$ present but not fully formed $=1 ;<50 \%$ present or completely absent $=2$. Simon et al and Barkovich et al considered the appearance of structures other than the deep gray nuclei to be important in describing HPE (eg, the corpus callosum and the Sylvian fissures). The corpus callosum can be assessed quite easily on iuMR, but the calculation of a Sylvian angle as suggested by Barkovich et $\mathrm{al}^{9}$ is not practicable in second trimester cases. It was even difficult in third trimester cases, giving nonreproducible assessments, so we did not include it in our approach to analyzing the images.

Despite those limitations, our data show a close correlation between the DeMyer classification used in this study and the se- verity score calculated in our study, but there were overlaps, particularly between the semilobar and lobar cases. For example, 4 cases had a severity score of 10 , but 2 were classified as lobar, and 2 , as semilobar. We believe that in most cases, the DeMyer classification is the most appropriate mechanism of relaying information based on the ease of assessment and the accessibility of the terms to non-neurology clinicians. There are cases in the midrange of severity or unusual cases (such as the case in Fig 6), however, in which the more detailed anatomic information that forms the severity score would be useful.

A further weakness of our study was the change in imaging protocols that occurred during the 15-year period of data collection, but this is inevitable when a new and rapidly changing technique, such as iuMR, is used to study rare pathology. This point is well-illustrated when trying to image the fetal hypothalamus with iuMR, which, as stressed by Simon et al, ${ }^{8,10}$ is an important anatomic feature of HPE in their publications concerning pediatric HPE. ${ }^{9}$ Those authors were the first to bring the MIHF variety of HPE to the wider attention of the imaging community, and they highlighted the importance of involvement of the hypothalamus in distinguishing standard ventral HPE and MIHF, which affects the dorsal structures. They noted some degree of noncleavage of the hypothalamus in all 56 cases of standard ventral HPE that could be assessed. In contrast, the hypothalamus was normally 
separated in all 21 of their cases of MIHF. They consider the hypothalamus to be a key structure in the assessment of pediatric $\mathrm{HPE}$, and our findings from iuMR of the fetus concur with that view.

The fetal hypothalamus, however, is a very small structure, and reviewing the cases from the earlier part of recruitment highlighted a potential problem. At the time when only singleshot fast spin-echo sequences with 5-mm-thick sections were used, we thought that there was a real risk of overcalling hypothalamic fusion because of partial volume effects on the coronal images. Improvements in gradient performance and software allowed 2- to 3-mm partitions, but the most significant advance in our experience has been the introduction of $3 \mathrm{D}$ balanced steady-state imaging. Those sequences produce images with high signal-to-noise and permit fast imaging times due to a TR that is shorter than the T2 of the tissue in study. The $\mathrm{T} 1 / \mathrm{T} 2$ ratios, rather than the $\mathrm{T} 1 / \mathrm{T} 2$ differences, are responsible for image contrast and can be varied by changing the flip angle and are independent of the TR. The partition thickness on our current examinations can be reduced to $1.8 \mathrm{~mm}$ without major loss of signal/noise, and review of the base data of those acquisitions is very helpful when imaging the fetal brain. The production of individualized surface representations of the brain from the 3D datasets can also be helpful diagnostically but is perhaps more useful when explaining the findings to clinical colleagues and parents.

\section{CONCLUSIONS}

We have shown that all aspects of the imaging diagnosis of fetal HPE are improved when antenatal US is supplemented with iuMR of the fetal brain. Advantages were found in confirming or refuting the diagnosis of HPE, classifying the severity of HPE, and recognizing associated brain abnormalities. All of these factors should improve the quality of information given to parents in terms of anatomic diagnosis and prognosis.

Disclosures: Paul D. Griffiths-RELATED: Other: GE Healthcare (research partnership)*; UNRELATED: Medical Research Council,* National Institute for Health Research-Health Technology Assessment, ${ }^{*}$ Wellcome Trust, ${ }^{\star}$ GE Healthcare, ${ }^{*}$ Comments: awarded current grants. *Money paid to the institution.

\section{REFERENCES}

1. Griffiths PD, Reeves J, Larroche JC, et al. Atlas of Fetal and Postnatal Brain MR Imaging. Philadelphia: Mosby; 2010

2. Jarvis DA, Armitage P, Dean A, et al. Surface reconstructions of foetal brain abnormalities using ultrafast steady state 3D acquisitions. Clin Radiol 2014;69:1084-91 CrossRef Medline

3. Griffiths PD, Jarvis D, McQuillan H, et al. MRI of the foetal brain using a rapid 3D steady-state sequence. Br J Radiol 2013;86: 20130168 CrossRef Medline

4. Chen H. Atlas of Genetic Diagnosis and Counseling. Totowa, NJ: Humana Press; 2006:493-501

5. Griffiths PD. Protocol 11PRT/2491: Magnetic resonance imaging to enhance the diagnosis of fetal developmental brain abnormalities in utero (MERIDIAN) (1SRCTN27626961). 2012. www.thelancet. com/protocol-reviews/11PRT-2491. Accessed October 15, 2015

6. DeMyer W, Zeman W. Alobar holoprosencephaly (arhinencephaly) with median cleft lip and palate: clinical, electroencephalographic and nosologic considerations. Confin Neurol 1963;23: 1-36 Medline

7. DeMyer W. Holoprosencephaly (cyclopia-arrhinencephaly). In: Myrianthopoulos N, ed. Malformations. New York: Elsevier, 1987: 225-44

8. Simon EM, Hevner R, Pinter JD, et al. Assessment of the deep gray nuclei in holoprosencephaly. AJNR Am J Neuroradiol 2000;21: 1955-61 Medline

9. Barkovich AJ, Simon EM, Clegg NJ, et al. Analysis of the cerebral cortex in holoprosencephaly with attention to the Sylvian fissures. AJNR Am J Neuroradiol 2002;23:143-50 Medline

10. Simon EM, Hevner RF, Pinter JD, et al. The middle interhemispheric variant of holoprosencephaly. AJNR Am J Neuroradiol 2002;23: 151-56 Medline

11. Rossi AC, Prefumo F. Additional value of fetal magnetic resonance imaging in the prenatal diagnosis of central nervous system anomalies: a systematic review of the literature. Ultrasound Obstet Gynecol 2014;44:388-93 CrossRef Medline

12. Griffiths PD, Batty R, Reeves MJ, et al. Imaging the corpus callosum, septum pellucidum and fornix in children: normal anatomy and variations of normality. Neuroradiology 2009;51:337-45 CrossRef Medline

13. Griffiths PD, Batty R, Connolly DA, et al. Effects of failed commissuration on the septum pellucidum and fornix: implications for fetal imaging. Neuroradiology 2009;51:347-56 CrossRef Medline

14. Barkovich AJ, Raybaud CA. Congenital malformations of the brain and skull. In: Barkovich AJ, Raybaud CA, eds. Pediatric Neuroimaging. 5th ed. Philadelphia: Wolters Kluwer Health/Lippincott Williams \& Wilkins; 2012:367-568 